\title{
The breeding biology of the Coal Tit Periparus ater in South Central Sweden
}

\author{
Svartmesens Periparus ater häckningsbiologi i Mellansverige
}

KARL GUSTAV SCHÖLIN \& HANS KÄLLANDER

\begin{abstract}
In a nestbox study started in 1948 and still ongoing, the number of breeding Coal Tits Periparus ater was always low. Here we present data on time of egg laying and its advancement during the last three decades, clutch size, fledging success and proportion of second broods. Laying dates varied between 18 April and 10 May, with an overall mean of 5 May, and advanced by 0.36 days per year during the second half of the study. Mean clutch size was 8.55 (first clutches). A mean of 7.25 young fledged (all first broods) or 7.78 (failed first broods excluded).

Second broods (12.6\%) were often not checked but seven clutches had a mean size of 7.23 eggs and fledged 6.71 young.

Karl Gustav Schölin, Kyrkvärdsg. 10, SE-702 84 Örebro, Sweden.E-mail:kalle.scholin@gmail.com

Hans Källander, Department of Biology (Ecology), Lund University, Ecology Building, SE-223 62 Lund, Sweden. E-mail:hans.kallander@telia.com
\end{abstract}

\section{Abstract}

Received 10 September 2013, Accepted 7 November 2013, Editor R. Ekblom

\section{Introduction}

Remarkably little seems to have been written on the breeding biology of the Coal Tit Periparus ater in Sweden. Durango (1946) discussed its habitat distribution, claiming that the species which is normally associated with coniferous forest occasionally can be found in predominantly deciduous forest. He also showed that a high proportion of nests was found in cracks in walls and rocks, among tree roots, etc., but gave no data on the Coal Tit's basic breeding biology, such as laying time, clutch size and fledging success. Here we present such data for the Coal Tit from a long-term study of small passerines breeding in nestboxes in a mixed coniferous-deciduous forest near Örebro, South Central Sweden. Although the yearly number of Coal Tits in the boxes was low, the length of the study ( 65 years) means that the total number of first clutches nonetheless exceeds 100. Our hope is that others running nestbox studies in other parts of Sweden will be stimulated to present their results on the species.

\section{Study area and methods}

The study area some $5 \mathrm{~km}$ SE of Örebro, South Central Sweden $\left(59^{\circ} 14^{\prime} \mathrm{N}, 15^{\circ} 13^{\prime} \mathrm{E}\right)$ has been described earlier as have the methods used (Schölin 2009, Schölin \& Källander 2011, 2012). The study was started in 1948 and is still ongoing. The number of nestboxes varied between 100 (highest number) in 1951 and 58 (lowest number) in 1961. They were of a size suitable for Great Tit Parus major and Pied Flycatcher Ficedula hypoleuca, placed at a height of c. $2.5 \mathrm{~m}$ and were checked about once a week during the breeding period with the exception of 1964 and 1965 when the boxes were not monitored regularly. First egg dates were back-calculated from the number of eggs found in the nest assuming the laying of one egg each day. Here we present data on the Coal Tit collected during 1949-2012 (no Coal Tits bred in the starting year).

Possible trends in laying date over the period and the relationship between laying date and mean April temperature were analysed by linear regressions. Temperature data were taken from the Swedish Meteorological and Hydrological Institute's (SMHI) published reports and are from the meteorological station in Örebro. It should be noted that mean monthly temperature is a rather rough measure of the temperature that influences spring phenology and may be responsible for some of the scatter in the regressions. In years when two 
or more Coal Tits bred in the boxes the mean laying date was used; in years with a single breeding pair, the laying date of that pair was used. Statistical tests were run on SYSTATтM (Wilkinson 1987).

\section{Results}

\section{Laying dates}

The number of Coal Tit pairs that bred in the boxes varied from none in eight of the years to six in 1988. The start of laying was earliest in 2007 (18 April) and latest in 2003 (10 May), with a mean for the whole period of 5 May $(\mathrm{N}=55$, Figure 1). Despite considerable scatter in the data points, laying was negatively associated with mean April temperature $(\mathrm{b}=-1.439, \mathrm{t}=-4.150, \mathrm{P}<0.001$, Figure 2). However, laying dates showed no trend during the first half of the study $(b=0.061, t=0.935$,

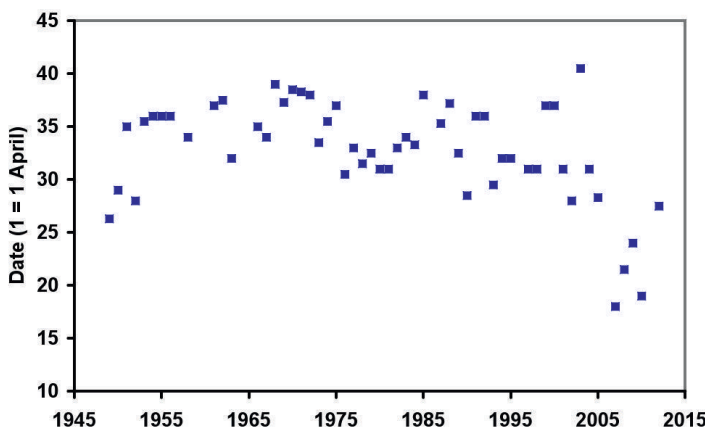

Figure 1. Mean laying dates of Coal Tits during 1949 to 2012 near Örebro, South Central Sweden.

Medelläggdatum för svartmesar c. $5 \mathrm{~km}$ SO om Örebro under åren $1949-2012$.

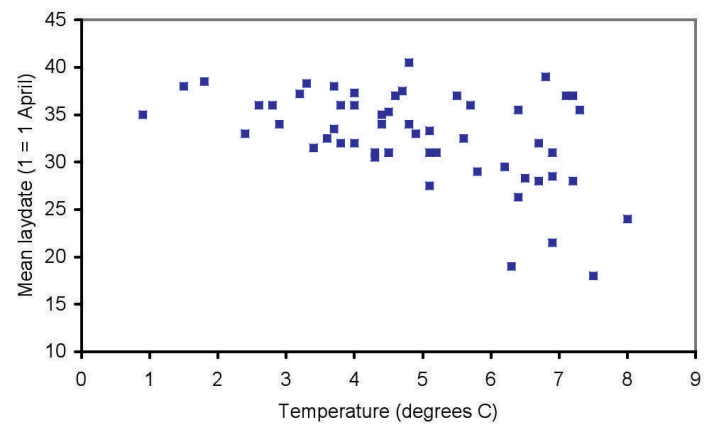

Figure 2. Mean Coal Tit laying dates plotted against mean April temperatures, 1949 to 2012, near Örebro, South Central Sweden.

Medelläggdatum för svartmesar c. $5 \mathrm{~km}$ SO om Örebro 1949 - 2012 plottade mot medeltemperaturen under april dessa år.

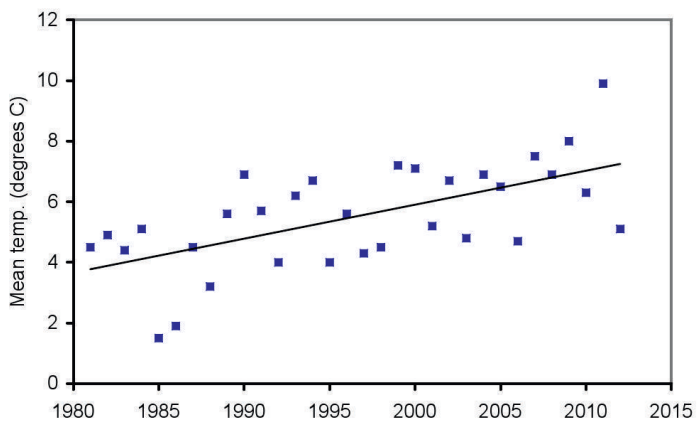

Figure 3. Mean April temperatures at Örebro, South Central Sweden during $1981-2012$.

Medeltemperaturen i Örebro åren 1981 - 2012.

$\mathrm{N}=27, \mathrm{P}=0.359)$, but did so during the second half $(\mathrm{b}=-0.348, \mathrm{t}=-3.644, \mathrm{~N}=28, \mathrm{P}=0.001)$ in agreement with the trend of increasing April temperatures during 1981 to $2012(\mathrm{~b}=0.112, \mathrm{t}=4.215$, $\mathrm{P}<0.001$, Figure 3).

\section{Clutch size}

The size of full-laid first clutches varied from six to 11 eggs (Figure 4, Appendix 1) with a mean of 8.55 \pm 0.94 (SD) eggs $(\mathrm{N}=103$ clutches $)$. Mean clutch sizes were almost identical during the first and the second half of the study period, $8.62 \pm 0.54$ vs 8.77 \pm 0.93 . Second clutches were relatively rare, only 13 compared with the 103 first clutches (12.6\%), and were mostly laid in the same nestbox almost

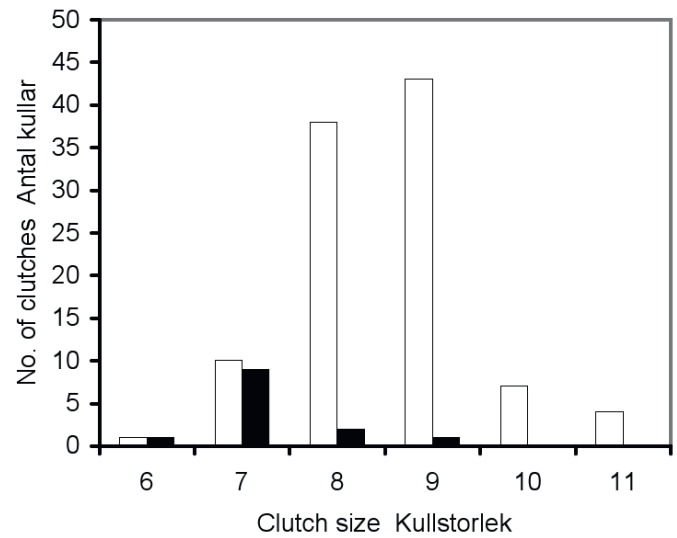

Figure 4. The distribution of clutch sizes of Coal Tits near Örebro, South Central Sweden (open bars: first clutches, filled bars: second clutches).

Fördelningen av svartmesens kullstorlekar i studieområdet c.5 km SO om Örebro (öppna staplar: förstakullar, fyllda staplar: andrakullar). 
immediately after the first brood fledged. They had a mean size of $7.23 \pm 0.73 \mathrm{eggs}$, i.e. they were about 1.5 eggs smaller than first clutches.

\section{Fledging rate}

Seven first broods $(6.8 \%)$ failed to produce fledglings. Those that did fledged a mean of $7.78 \pm 1.24$ young or $91 \%$ of the number of eggs laid. Corresponding figures for all first broods, i.e. including total losses, was $7.25 \pm 2.30$ fledged young or c. $85 \%$ of eggs laid (Appendix 2). The cause of total loss was predation by Pine Marten Martes martes ( 2 cases), and by Great Spotted Woodpecker Dendrocopos major (3 cases). In one nestbox the female was found dead on her eggs and in another one the nestbox had been taken over by a Pied Flycatcher, probably after the Coal Tid had deserted its clutch. Fledging rate was not checked for all second broods but reached a mean of 6.71 young fledged $(\mathrm{N}=7, \mathrm{SD}=1.25)$.

\section{Discussion}

Like the Blue Tits Cyanistes caeruleus in the same nestbox study (Schölin \& Källander 2012), Coal Tits responded to the increasing April temperatures during the second half of the study period by laying earlier. This contrasts with the results of an English study of Coal Tits breeding in a deciduous habitat (Goodenough et al. 2010). While these authors found that Great Tit, Blue Tit and European Nuthatch Sitta europaea had advanced laying during the last circa two decades, the Coal Tit had not done so. The authors had no explanation for this deviating pattern. In our data there was a somewhat larger scatter in the Coal Tit data (SD 5.75) during the latter part of the study than in those of the Blue Tit (SD 4.57). The reason for this is unknown, but one possibility could be that Coal Tits sometimes may have got their selected nestbox usurped by Great Tits or Blue Tits and been forced to find a new empty box before being able to start building a nest. A few examples of Coal Tits being evicted by larger species were given by Durango (1946). In a study in South Sweden $\left(57^{\circ} 58^{\prime} \mathrm{N}, 14^{\circ} 48^{\prime} \mathrm{E}\right)$, J.-E. Nilsson (pers.comm.) found the mean laying date to be 25 April $(\mathrm{N}=18)$ with a span from 19 April to 9 May during the period 1986-2004. The latest laying date was in 2003, just as in the present study. In our study the laying date for the same period was 3 May.

The mean size of first clutches of the Coal Tits in the present study, c.8.6 eggs, is similar to sizes reported from larch Larix sp. and spruce Abies alba forests in lowland Germany (Glutz \& Bauer 1993) but is smaller than that reported from pine forest in England, 9.1 - 10.5 eggs (Lack 1966). In 28 first

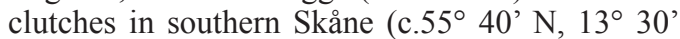
E), the mean clutch size was $9.50 \pm 0.84$ (Jan-Åke Nilsson pers.comm.).

As in the English study (Lack 1966), Coal Tits had a rather high fledging success for first broods with $85 \%$ of eggs laid resulting in fledged young. The cirka 1.5 fewer eggs in second broods found in the present study agrees with findings from other areas of Europe (Glutz \& Bauer 1993).

\section{Acknowledgements}

We thank Jan-Eric Nilsson and Jan-Åke Nilsson for allowing us to use their data on laying dates and clutch sizes, respectively.

\section{References}

Durango, S. 1946. Habitat and nesting sites of the Coal-Tit (Parus ater ater L.) Vår Fågelvärld 5: 1-9. (In Swedish with summary in English.)

Glutz, U.N. \& Bauer, K.M. 1993. Handbuch der Vögel Mitteleuropas. Band 13/I. Aula-Verlag, Wiesbaden.

Goodenough, A.E., Hart, A.G. \& Stafford, R. 2010. Is adjustment of breeding phenology keeping pace with the need for change? Linking observed response in woodland birds to changes in temperature and selection pressure. Climatic Change 102: 687-697.

Lack, D. 1966. Population studies of birds. Clarendon Press, Oxford.

Schölin, K.G. 2009. A long-term study of the breeding biology of the Great Tit Parus major in a mixed forest in South Central Sweden. Ornis Svecica 19: 222-232. (In Swedish with summary in English.)

Schölin, K. G. \& Källander, H. 2011. A 64-year study of a Pied Flycatcher Ficedula hypoleuca population. Ornis Svecica 21: 79-91.

Schölin, K.-G. \& Källander, H. 2012. A Blue Tit Cyanistes caeruleus population: its recent increase and breeding data. Ornis Svecica 22: 19-24.

Wilkinson, L. 1987. The system for statistics. SYSTAT Inc., Evanston.

\section{Sammanfattning}

Det tycks inte finnas några publicerade uppsatser från Sverige om svartmesens Periparus ater häckningsbiologi utöver några omnämnanden om boplatsval (Durango 1946). Här presenteras data om läggningsstart, kullstorlek och antal flygga ungar hos svartmes från en 65-årig holkstudie bedriven strax SO om Örebro. För en beskrivning av studieområde och metod hänvisas till Schölin (2009) och 
Schölin \& Källander $(2011,2012)$. Totalt omfattar materialet 103 förstakullar, men svartmes häckade inte varje år i holkarna.

Medeldatum för läggstarten var 5 maj, med 18 april som tidigaste datum och 10 maj som det senaste (Figur 1). Under studiens första hälft fanns ingen trend i läggdatum, medan läggstarten under dess andra hälft inträffade i medeltal en dryg tredjedels dag tidigare för vart år (Figur 2), i samstämmighet med en statistiskt signifikant trend mot högre apriltemperaturer $\left(0,11^{\circ} \mathrm{C}\right.$ per år, Figur 3$)$.

Medelkullstorleken för förstakullar var 8,55 ägg (Appendix 1, Figur 4) och var nästan identisk mellan den första och andra halvan av studien. Andrakullarna, totalt 13 (12,6\% av antalet förstakullar) var c. 1,5 ägg mindre (medel 7,23).

Totalt blev i medeltal 7,25 förstakullsungar flygga eller 7,78 om sju kullar som helt misslyckades utesluts. Det innebär att c. 85 respektive $91 \%$ av lagda ägg resulterade i flygga ungar (Appendix 2). Av de misslyckade kullarna prederades två av mård och tre av större hackspett, i en holk låg honan död på äggen och en kull överbyggdes av svartvit flugsnappare, sannolikt efter det att svartmesen övergett boet. Andrakullars framgång kontrollerades inte alla år, men de sju kullar som följdes producerade 6,7 flygga ungar.
Medeldatum för förstakullarnas läggning var något senare (5 maj) än vad J.-E. Nilsson (pers. comm.) fann vid Linderås sydväst om Tranås åren 1996-2004 (25 april). För dessa år var medeldatum 3 maj utanför Örebro. I en lövskogsbiotop i England fann Goodenough et al. (2010), ingen tidigareläggning hos svartmes trots ökande temperatur under perioden och i motsats till vad fallet var hos talgoxe Parus major, blåmes Cyanistes caeruleus och nötväcka Sitta europaea. Författarna fann ingen förklaring till denna skillnad mellan arterna.

Medelkullstorleken i denna studie är jämförbar med värden från låglandsbarrskogar i Tyskland (Glutz \& Bauer 1993), men är mindre än motsvarande värden från England, 9,1-10,5 ägg (Lack 1966) och också mindre än vad som noterats i södra Skåne, 9,5 ägg (J.-Å. Nilsson pers. comm.). Andrakullarnas cirka 1,5 färre ägg överensstämmer väl med vad som noterats $i$ andra europeiska studier.

Förstakullarna hade god framgång. Av äggen resulterade $85 \%$ i flygga ungar, när även misslyckade kullar är inräknade, och hela $91 \%$ om bara kullar som producerat flygga ungar räknas in (Appendix 2). De sju andrakullar som följdes, producerade 6,7 flygga ungar, medan häckningsutfallet inte följdes i de återstående sex andrakullarna. 
Appendix 1. Number of clutches of different size in Coal Tits Periparus ater at Lövsätter, near Örebro,South Central Sweden during 1949 - 2012.

Antal svartmeskullar av olika storlek vid Lövsätter SO om Örebro under åren 1949 - 2012.

\begin{tabular}{|c|c|c|c|c|c|c|c|c|c|}
\hline \multirow{2}{*}{$\begin{array}{l}\text { Year } \\
\text { År }\end{array}$} & \multicolumn{6}{|c|}{ Clutch size Kullstorlek } & \multirow{2}{*}{$\begin{array}{l}\text { No.of clutches } \\
\text { Antal kullar }\end{array}$} & \multirow{2}{*}{$\begin{array}{l}\text { No. of eggs } \\
\text { Antal ägg }\end{array}$} & \multirow{2}{*}{$\begin{array}{l}\text { Mean clutch size } \\
\text { Medelkullstorlek }\end{array}$} \\
\hline & 6 & 7 & 8 & 9 & 10 & 11 & & & \\
\hline 1949 & & & 1 & 1 & 1 & & 3 & 27 & 9.00 \\
\hline 1950 & & & 1 & & & & 1 & 8 & 8.00 \\
\hline 1951 & & & & 1 & & & 1 & 9 & 9.00 \\
\hline 1952 & & & & 1 & & & 1 & 9 & 9.00 \\
\hline 1953 & & & 2 & & & & 2 & 16 & 8.00 \\
\hline 1954 & & & 1 & & & & 1 & 8 & 8.00 \\
\hline 1955 & & & & 1 & & & 1 & 9 & 9.00 \\
\hline 1956 & & & & 1 & & & 1 & 9 & 9.00 \\
\hline 1957 & & & & & & & 0 & - & - \\
\hline 1958 & & & & 1 & & & 1 & 9 & 9.00 \\
\hline 1959 & & & & & & & 0 & & - \\
\hline 1960 & & & & & & & 0 & - & - \\
\hline 1961 & & & & 1 & & & 1 & 9 & 9.00 \\
\hline 1962 & & & 1 & 1 & & & 2 & 17 & 8.50 \\
\hline 1963 & & & & 1 & & & 1 & 9 & 9.00 \\
\hline 1964 & & & & & & & 4* & - & - \\
\hline 1965 & & & & & & & 4* & - & - \\
\hline 1966 & & 1 & 1 & & 1 & & 3 & 25 & 8.33 \\
\hline 1967 & & & & 2 & & & 2 & 18 & 9.00 \\
\hline 1968 & & & 1 & 2 & & & 3 & 26 & 8.67 \\
\hline 1969 & & & 2 & 1 & & & 3 & 25 & 8.33 \\
\hline 1970 & & & 2 & & & & 2 & 16 & 8.00 \\
\hline 1971 & & 1 & & 2 & & & 3 & 25 & 8.33 \\
\hline 1972 & & & 1 & & & & 1 & 8 & 8.00 \\
\hline 1973 & & & 1 & 1 & & & 2 & 17 & 8.50 \\
\hline 1974 & & & & 2 & & & 2 & 18 & 9.00 \\
\hline 1975 & & & & & 1 & & 1 & 10 & 10.00 \\
\hline 1976 & & 1 & 1 & & & & 2 & 15 & 7.50 \\
\hline 1977 & & & & 1 & & & 1 & 9 & 9.00 \\
\hline 1978 & & & 1 & 1 & & & 2 & 17 & 8.50 \\
\hline 1979 & & 1 & & 1 & & & 2 & 16 & 8.00 \\
\hline 1980 & & & & 1 & & & 1 & 9 & 9.00 \\
\hline 1981 & & 1 & & 1 & & & 2 & 16 & 8.00 \\
\hline 1982 & & & 1 & 1 & & & 2 & 17 & 8.50 \\
\hline 1983 & & & 1 & 1 & & & 2 & 17 & 8.50 \\
\hline 1984 & & & 1 & 2 & & & 3 & 26 & 8.67 \\
\hline 1985 & & & 1 & & & & 1 & 8 & 8.00 \\
\hline 1986 & & & & & & & 0 & & - \\
\hline 1987 & & 1 & 1 & 1 & & & 3 & 24 & 8.00 \\
\hline
\end{tabular}


Appendix 1, continued

\begin{tabular}{|c|c|c|c|c|c|c|c|c|c|}
\hline 1988 & & 1 & 4 & & 1 & & 6 & 49 & 8.17 \\
\hline 1989 & & & 1 & 1 & & & 2 & 17 & 8.50 \\
\hline 1990 & & & 3 & 1 & & & 4 & 33 & 8.25 \\
\hline 1991 & & & 2 & 1 & & & 3 & 25 & 8.33 \\
\hline 1992 & & & 1 & 1 & & & 2 & 17 & 8.50 \\
\hline 1993 & & & 2 & & & & 2 & 16 & 8.00 \\
\hline 1994 & & & & & 1 & & 1 & 10 & 10.00 \\
\hline 1995 & & & & 1 & & & 1 & 9 & 9.00 \\
\hline 1996 & & & & & & & 0 & - & - \\
\hline 1997 & & & 1 & 1 & & & 2 & 17 & 8.50 \\
\hline 1998 & & & & & & 1 & 1 & 11 & 11.00 \\
\hline 1999 & & 1 & & & 1 & & 2 & 17 & 8.50 \\
\hline 2000 & & & & 1 & & & 1 & 9 & 9.00 \\
\hline 2001 & & & & 1 & & & 1 & 9 & 9.00 \\
\hline 2002 & 1 & & & 1 & & & 2 & 15 & 7.50 \\
\hline 2003 & & & 2 & & & & 2 & 16 & 8.00 \\
\hline 2004 & & & & 1 & & & 1 & 9 & 9.00 \\
\hline 2005 & & 1 & 1 & 1 & & & 3 & 24 & 8.00 \\
\hline 2006 & & & & & & & 0 & - & - \\
\hline 2007 & & & & 1 & & 1 & 2 & 20 & 10.00 \\
\hline 2008 & & 1 & & 1 & & & 2 & 16 & 8.00 \\
\hline 2009 & & & & & & 1 & 1 & 11 & 11.00 \\
\hline 2010 & & & & 1 & & & 1 & 9 & 9.00 \\
\hline 2011 & & & & & & & 0 & - & - \\
\hline 2012 & & & & & 1 & 1 & 2 & 21 & 10.50 \\
\hline Total & 1 & 10 & 38 & 43 & 7 & 4 & 103 & 881 & 8.55 \\
\hline
\end{tabular}

* Clutch size not checked Kullstorlek ej kontrollerad 
Appendix 2. Number of Coal Tit broods from which 0, 1, 2, 3, etc. young fledged during 1949 - 2012 at Lövsätter near Örebro in South Central Sweden.

Antal kullar av svartmes från vilka 0, 1, 2, 3, etc. ungar blev flygga vid Lövsätter utanför Örebro under åren $1949-2012$.

\begin{tabular}{|c|c|c|c|c|c|c|c|c|c|c|c|c|c|c|c|}
\hline \multirow{2}{*}{$\begin{array}{l}\text { Year } \\
\text { Ar }\end{array}$} & \multicolumn{12}{|c|}{ No. of young fledged Antal flygga ungar } & \multirow{2}{*}{$\begin{array}{l}\text { No. fledged } \\
\text { Antal flygga }\end{array}$} & \multirow{2}{*}{$\begin{array}{l}\text { No. broods } \\
\text { Antal kullar }\end{array}$} & \multirow{2}{*}{$\begin{array}{c}\text { Mean fledged } \\
\text { Medeltal flygga }\end{array}$} \\
\hline & 0 & 1 & 2 & 3 & 4 & 5 & 6 & 7 & 8 & 9 & 10 & 11 & & & \\
\hline 1949 & 2 & & & & & & & & & & 1 & & 10 & 3 & 3.33 \\
\hline 1950 & & & & & & & & & 1 & & & & 8 & 1 & 8.00 \\
\hline 1951 & & & & & & & & & 1 & & & & 8 & 1 & 8.00 \\
\hline 1952 & & & & & & & & & 1 & & & & 8 & 1 & 8.00 \\
\hline 1953 & & & & & & & 1 & 1 & & & & & 13 & 2 & 6.50 \\
\hline 1954 & & & & & & & & 1 & & & & & 7 & 1 & 7.00 \\
\hline 1955 & & & & & & & & 1 & & & & & 7 & 1 & 7.00 \\
\hline 1956 & & & & & & & & & & 1 & & & 9 & 1 & 9.00 \\
\hline 1957 & & & & & & & & & & & & & - & - & \\
\hline 1958 & & & & & & & & & 1 & & & & 8 & 1 & 8.00 \\
\hline 1959 & & & & & & & & & & & & & & - & \\
\hline 1960 & & & & & & & & & & & & & - & - & \\
\hline 1961 & & & & & & & & & 1 & & & & 8 & 1 & 8.00 \\
\hline 1962 & & & & & & & & & 1 & 1 & & & 17 & 2 & 8.50 \\
\hline 1963 & & & & & & & & & & 1 & & & 9 & 1 & 9.00 \\
\hline 1964 & & & & & & & & & & & & & & $(4)^{*}$ & \\
\hline 1965 & & & & & & & & & & & & & & $(4)^{*}$ & \\
\hline 1966 & & & & & & & & 1 & 2 & & & & 23 & 3 & 7.67 \\
\hline 1967 & & & & & & & & 1 & & 1 & & & 16 & 2 & 8.00 \\
\hline 1968 & & & & & & & & & 3 & & & & 24 & 3 & 8.00 \\
\hline 1969 & & & & & & & 1 & 1 & & 1 & & & 22 & 3 & 7.33 \\
\hline 1970 & & & & & & & & 1 & 1 & & & & 15 & 2 & 7.50 \\
\hline 1971 & & & & & 1 & & & 1 & 1 & & & & 19 & 3 & 6.33 \\
\hline 1972 & & & & & & & & 1 & & & & & 7 & 1 & 7.00 \\
\hline 1973 & & & & & & & & 1 & & 1 & & & 16 & 2 & 8.00 \\
\hline 1974 & & & & & & & & 1 & 1 & & & & 15 & 2 & 7.50 \\
\hline 1975 & & & & & & & & & 1 & & & & 8 & 1 & 8.00 \\
\hline 1976 & & & & & & & 1 & & 1 & & & & 14 & 2 & 7.00 \\
\hline 1977 & & & & & & & & & 1 & & & & 8 & 1 & 8.00 \\
\hline 1978 & & & & & & & & 1 & 1 & & & & 15 & 2 & 7.50 \\
\hline 1979 & & & & & & & & 2 & & & & & 14 & 2 & 7.00 \\
\hline 1980 & & & & & & & & & 1 & & & & 8 & 1 & 8.00 \\
\hline 1981 & & & & & & & 1 & & & 1 & & & 15 & 2 & 7.50 \\
\hline 1982 & & & & & & & & 1 & & 1 & & & 16 & 2 & 8.00 \\
\hline 1983 & & & & & & & & 1 & 1 & & & & 15 & 2 & 7.50 \\
\hline 1984 & & & & & & & & 1 & 1 & 1 & & & 24 & 3 & 8.00 \\
\hline 1985 & & & & & & & & & 1 & & & & 8 & 1 & 8.00 \\
\hline 1986 & & & & & & & & & & & & & - & - & \\
\hline
\end{tabular}


Appendix 2, continued

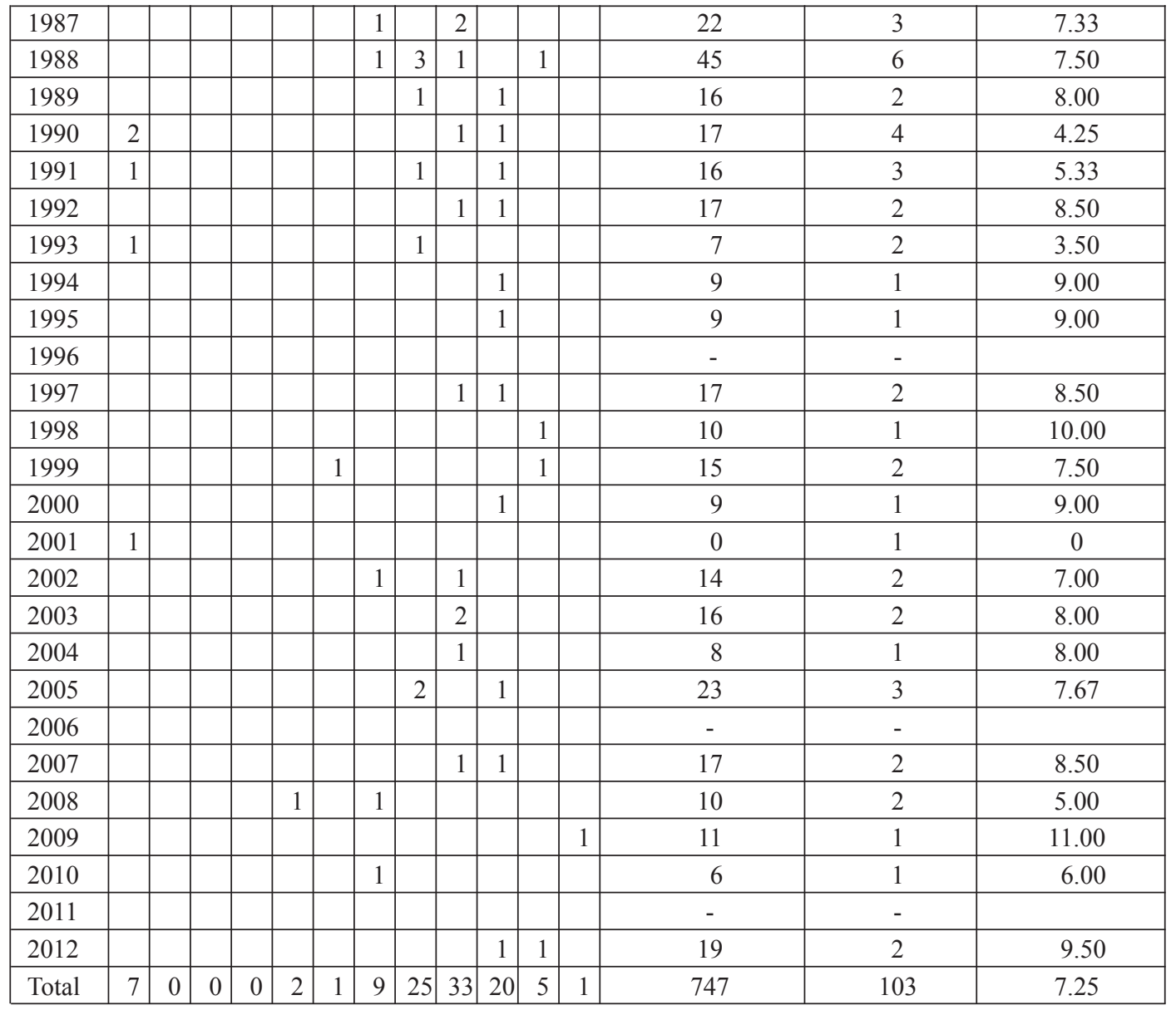

* Brood success not checked Framgång ej kontrollerad 